\title{
The rôle of the Berry Phase in Dynamical Jahn-Teller Systems
}

\author{
Nicola Manini \\ European Synchrotron Radiation Facility, B.P. 220, F-38043 Grenoble Cédex, France \\ Paolo De Los Rios \\ Institut de Physique Théorique, Université de Fribourg, 1700-CH Fribourg, Switzerland
}

(June 11, 2018)

\begin{abstract}
The presence/absence of a Berry phase depends on the topology of the manifold of dynamical JahnTeller potential minima. We describe in detail the relation between these topological properties and the way the lowest two adiabatic potential surfaces get locally degenerate. We illustrate our arguments through spherical generalizations of the linear $T \otimes h$ and $H \otimes h$ cases, relevant for the physics of fullerene ions. Our analysis allows us to classify all the spherical Jahn-Teller systems with respect to the Berry phase. Its absence can, but does not necessarily, lead to a nondegenerate ground state.
\end{abstract}

\section{INTRODUCTION}

The traditional field of degenerate electron-lattice interactions (Jahn-Teller effect) in molecules and impurity centers in solids [1,2] has attracted new interest in recent years, excited by the realization of new systems which call for a revision of a number of commonly accepted beliefs. A whole range of icosahedral molecular systems including $\mathrm{C}_{60}$ ions and some higher fullerenes, thanks to the rich structure of the symmetry group, are characterized by up to fivefold-degenerate representations of the electronic and vibrational states of the isolated molecule/ion. New Jahn-Teller (JT) systems have therefore been considered theoretically, 24 with intriguing features. [4 A particularly surprising property has been demonstrated recently: the possibility of a symmetry switch of the molecular ground state [7] 8$]$, connected to the absence of Berry phase in the coupled dynamics of electrons and vibrations.

As it is well known, the molecular symmetry, reduced in the static JT effect with the splitting of the electronicstate degeneracy, is restored when the coherent tunneling between equivalent distortions is considered, in the dynamical Jahn-Teller (DJT) effect. In this context it was commonly accepted an empirical "symmetry conservation rule", sometimes referred to as "Ham's theorem", stating that the symmetry of the vibronic DJT ground state, at all coupling strengths, remains the same as that of the electronic multiplet prior to coupling: [2] all linear JT systems known till a few years ago, for singleelectron occupancy, systematically satisfy this empiric rule. It was understood recently that this phenomenon, not automatically implied by the DJT physics, is in reality a fingerprint of a Berry phase [9] in the entangled electronic-phononic dynamics. [4,7, 10 Consequently, this geometrical phase seemed a universal feature of the DJT systems.
It came unexpected the discovery of the first dynamical JT system without Berry phase, showing a nondegenerate ground state in the strong-coupling limit. [7, \&] This is the model that in spherical symmetry is indicated as $\mathcal{D}^{(2)} \otimes d^{(2)}$, where electrons of angular momentum $L=2$ interact with vibrations also belonging to an $l=2$ representation. This system is relevant to the physics of fullerene ions $\mathrm{C}_{60}^{+}$, where the 5-fold degenerate electronic state has $H_{u}$ icosahedral label and the quadrupolar distortions correspond to some of the $h_{g}$ modes. [7] It has been shown both analytically and numerically that, for increasing coupling, a nondegenerate state in the vibronic spectrum moves down, to cross the 5 -fold ground state at some finite value of the coupling parameter, thus becoming the ground state at strong coupling [0],8].

In this work, we review the mechanism of the Berry phase in degenerate electron-vibration coupled systems, and uncover the detailed reason of the absence of this geometric phase in the $\mathcal{D}^{(2)} \otimes d^{(2)}$ system, hence drawing some natural generalizations. We structure the paper as follows. In Sect. [I], we introduce the basic JT Hamiltonian, along with the fundamental transformations to recast it in a semi-classical form. Section III reviews the mechanism responsible for the presence of the Berry phase in most DJT systems, and its consequences for the low-energy part of their spectrum. In Sect. IV, the mechanism allowing to get rid of the Berry phase in some systems is unveiled, and a class of models sharing this property is proposed. Section $\mathrm{V}$ reports some numerical results confirming and complementing the predictions of Sect. IV. In Sect. VI, we discuss possible generalizations of our model to higher-order couplings and discrete symmetry groups. Finally, conclusions are drawn in Sect. VII. 


\section{THE MODELS}

According to the general theory of the JT effect, [2] an $N$-fold degenerate electronic level corresponding to a representation $\Gamma$ of the molecular symmetry point group interacts with all the vibrational modes corresponding to representations $\{\Lambda\}$ contained in the symmetric part of the direct product of $\Gamma$ with itself. For simplicity, in this work we restrict to only one degenerate mode. The Hamiltonian for this " $\Gamma \otimes \Lambda$ " case reads:

$$
H=\hbar \omega \sum_{i=1}^{|\Lambda|} b_{i}^{\dagger} b_{i}+H_{\mathrm{e}-\mathrm{v}}
$$

where $b_{i}^{\dagger} / b_{i}$ are the creation/destruction operators for the harmonic vibrational mode component $i$, and $H_{\mathrm{e}-\mathrm{v}}$ is the interaction Hamiltonian, which, to linear order in the boson operators, writes

$$
H_{\mathrm{e}-\mathrm{v}}=\frac{1}{2} g \hbar \omega \sum_{i=1}^{|\Lambda|} \sum_{j, k=1}^{|\Gamma|}\left(b_{i} c_{j}^{\dagger} c_{k}\langle\Lambda i \mid \Gamma j \Gamma k\rangle+\text { h.c. }\right)
$$

$c_{j}$ is the fermion operator for orbital $j,\langle\Lambda i \mid \Gamma j \Gamma k\rangle$ are the Clebsch-Gordan coefficients of the symmetry group of the problem, and $g$ is the dimensionless coupling parameter. For the purpose of illustrating our analysis, it is particularly convenient to stick to the case in which the symmetry group is that of three-dimensional rotations, $\mathrm{SO}(3)$. In the following, therefore, we focus on the coupling of an electronic state of angular momentum $L$ (whose representation $\Gamma$ is indicated as $\mathcal{D}^{(L)}$ ) to a degenerate vibration of angular momentum $l$ (of representation $\left.\Lambda \equiv d^{(l)}\right)$. For this case, the Hamiltonian reads

$$
\begin{aligned}
H= & \hbar \omega \sum_{m=-l}^{l}\left\{b_{m}^{\dagger} b_{m}+\frac{g}{2} \sum_{k, k^{\prime}=-L}^{L}(-1)^{k^{\prime}}\right. \\
& {\left.\left[b_{m}^{\dagger}+(-1)^{m} b_{-m}\right] c_{k}^{\dagger} c_{k^{\prime}}\left\langle\operatorname{lm} \mid L k, L-k^{\prime}\right\rangle\right\}, }
\end{aligned}
$$

where some of the symmetries of the $S O(3)$ ClebschGordan coefficients are implied.

The Hamiltonian (3) is suitable for perturbation calculations (small $g$ values) and as a starting point for numerical diagonalization methods, such as the Lanczos technique. Yet, the Berry phase is a semiclassical concept, useful only in the medium/large $g$ regimes. Here it is convenient to switch to a real representation of the vibrational degrees of freedom.

We apply two unitary transformations, both on the electronic operators, and the vibrational ones. We define a new set of electronic operators, $\tilde{c}_{m}$ (and consequently their hermitian conjugate $\left.\tilde{c}_{m}^{\dagger}\right), m=-L, \ldots, L$ via the transformation

$$
\begin{aligned}
\tilde{c}_{0} & =c_{0} \\
\left(\begin{array}{c}
\tilde{c}_{m} \\
\tilde{c}_{-m}
\end{array}\right) & =\frac{\exp \frac{i \pi\left[(-)^{m}-1\right]}{4}}{\sqrt{2}}\left(\begin{array}{cc}
i & -i \\
1 & 1
\end{array}\right)\left(\begin{array}{c}
c_{m} \\
c_{-m}
\end{array}\right), m>0
\end{aligned}
$$

The second transformation expresses the $2 l+1$ boson operators $B_{m} \equiv b_{m}^{\dagger}+(-1)^{m} b_{-m}$ in terms of the Hermitean "coordinate" operators $q_{m}$ as follows:

$$
\begin{aligned}
B_{0} & =\sqrt{2} q_{0} \\
\left(\begin{array}{c}
B_{m} \\
B_{-m}
\end{array}\right) & =\left(\begin{array}{cc}
(-)^{m} & -i(-)^{m} \\
i & 1
\end{array}\right)\left(\begin{array}{c}
q_{m} \\
q_{-m}
\end{array}\right), m>0 .
\end{aligned}
$$

The remaining components $b_{m}^{\dagger}-(-1)^{m} b_{-m}$ can be expressed correspondingly in terms of the momentum operators $p_{m}$ conjugate to $q_{m}$. We stress that these transformations are by no means unique. Any further orthogonal transformation of the real coordinates, leads to equivalent results.

Eventually, the Hamiltonian operator (3) transforms into the form

$$
H=\frac{1}{2} \hbar \omega_{l} \sum_{M=-l}^{l}\left(p_{m}^{2}+q_{m}^{2}\right)+H_{\mathrm{e}-\mathrm{v}}
$$

with

$$
H_{\mathrm{e}-\mathrm{v}}=\frac{1}{\sqrt{2}} g \hbar \omega \sum_{m=-l}^{l} q_{m} \sum_{j, k=-L}^{L} \tilde{c}_{j}^{\dagger} V_{j, k}^{(m)} \tilde{c}_{k} .
$$

It is straightforward to compute the $(2 L+1) \times(2 L+1)$ coupling matrices $V^{(m)}$, for any value of $L$ and 1 . For brevity, we introduce the symbols $\gamma_{j, k}^{+} \equiv\langle l j+k \mid L j, L k\rangle$, $\gamma_{j, k}^{-} \equiv 0$. In this notation, the matrices for the $L=2$ case are:

$$
\begin{aligned}
& V^{(0)}=\left(\begin{array}{ccccc}
\gamma_{-2,2}^{+} & 0 & 0 & 0 & 0 \\
0 & -\gamma_{-1,1}^{+} & 0 & 0 & 0 \\
0 & 0 & \gamma_{0,0}^{+} & 0 & 0 \\
0 & 0 & 0 & -\gamma_{1,-1}^{+} & 0 \\
0 & 0 & 0 & 0 & \gamma_{2,-2}^{+}
\end{array}\right) \\
& V^{( \pm 1)}=\left(\begin{array}{ccccc}
0 & -\frac{\gamma_{-1,2}^{\mp}}{\sqrt{2}} & 0 & \frac{\gamma_{-1,2}^{ \pm}}{\sqrt{2}} & 0 \\
-\frac{\gamma_{2,-1}^{\mp}}{\sqrt{2}} & 0 & -\gamma_{0,1}^{\mp} & 0 & -\frac{\gamma_{2,-1}^{ \pm}}{\sqrt{2}} \\
0 & -\gamma_{1,0}^{\mp} & 0 & -\gamma_{1,0}^{ \pm} & 0 \\
\frac{\gamma_{2,-1}^{\mp}}{\sqrt{2}} & 0 & -\gamma_{0,1}^{ \pm} & 0 & -\frac{\gamma_{2,-1}^{\mp}}{\sqrt{2}} \\
0 & -\frac{\gamma_{-1,2}^{ \pm}}{\sqrt{2}} & 0 & -\frac{\gamma_{-1,2}^{\mp}}{\sqrt{2}} & 0
\end{array}\right) \\
& V^{( \pm 2)}=\left(\begin{array}{ccccc}
0 & 0 & -\gamma_{0,2}^{ \pm} & 0 & 0 \\
0 & -\frac{\gamma_{1,1}^{ \pm}}{\sqrt{2}} & 0 & \frac{\gamma_{1,1}^{\mp}}{\sqrt{2}} & 0 \\
\gamma_{0,2}^{ \pm} & 0 & 0 & 0 & -\gamma_{2,0}^{\mp} \\
0 & \frac{\gamma_{1,1}^{\mp}}{\sqrt{2}} & 0 & \frac{\gamma_{1,1}^{ \pm}}{\sqrt{2}} & 0 \\
0 & 0 & -\gamma_{0,2}^{\mp} & 0 & 0
\end{array}\right) \\
& V^{( \pm 3)}=\left(\begin{array}{ccccc}
0 & -\frac{\gamma_{1,2}^{\mp}}{\sqrt{2}} & 0 & -\frac{\gamma_{1,2}^{ \pm}}{\sqrt{2}} & 0 \\
-\frac{\gamma_{2,1}^{\mp}}{\sqrt{2}} & 0 & 0 & 0 & -\frac{\gamma_{2,1}^{ \pm}}{\sqrt{2}} \\
0 & 0 & 0 & 0 & 0 \\
-\frac{\gamma_{2,1}^{ \pm}}{\sqrt{2}} & 0 & 0 & 0 & \frac{\gamma_{2,1}^{\mp}}{\sqrt{2}} \\
0 & -\frac{\gamma_{1,2}^{ \pm}}{\sqrt{2}} & 0 & \frac{\gamma_{1,2}^{\mp}}{\sqrt{2}} & 0
\end{array}\right)
\end{aligned}
$$




$$
V^{( \pm 4)}=\left(\begin{array}{ccccc}
\frac{\gamma_{2,2}^{ \pm}}{\sqrt{2}} & 0 & 0 & 0 & -\frac{\gamma_{2,2}^{\mp}}{\sqrt{2}} \\
0 & 0 & 0 & 0 & 0 \\
0 & 0 & 0 & 0 & 0 \\
0 & 0 & 0 & 0 & 0 \\
-\frac{\gamma_{2,2}^{\mp}}{\sqrt{2}} & 0 & 0 & 0 & -\frac{\gamma_{2,2}^{ \pm}}{\sqrt{2}}
\end{array}\right)
$$

Note that the forms of $V^{(0)}, V^{( \pm 1)}$, and $V^{( \pm 2)}$ apply to the coupling to both $l=2$ and $l=4$ vibrons (of course, the numerical values of the coefficients are different), while the $V^{( \pm 3)}$ and $V^{( \pm 4)}$ matrices are relevant only for $l=4$.

For larger $L$ 's, the structure of the central $5 \times 5$ block (corresponding to indexes $j=-2$ to 2 ) of the corresponding coupling matrices is conserved, additional matrix elements being added externally in a simple way. For example, for any $L$ and $l$, the $V^{(0)}$ matrix is diagonal with matrix elements $V_{k, k}^{(0)}=(-1)^{k} \gamma_{k,-k}^{+}$. Similarly, if $l=2 \cdot L$ then the only nonzero elements of the $V^{( \pm l)}$ matrices are $V_{ \pm l, \pm l}^{(l)}= \pm \frac{\gamma_{l, l}^{ \pm}}{\sqrt{2}}$ and $V_{ \pm l, \mp l}^{(-l)}=-\frac{\gamma_{l, l}^{ \pm}}{\sqrt{2}}$.

As anticipated, the form (6.,7) of the Hamiltonian is suitable to a semiclassical treatment, in the spirit of the Born-Oppenheimer (BO) approximation, i.e. a factorization of the electronic "fast" dynamics from the "slow" distortions $q_{m}$, which are quantized as a second step. In this scheme, we briefly review the Berry phase mechanism, and its consequences on the factorized dynamics.

\section{THE BERRY PHASE}

The traditional BO scheme assumes that the electrons, moving much faster than the ions, follow adiabatically the ionic (vibrational) motion with the only effect of generating a potential energy for the vibrational motion. This approximation relies on separations between consecutive electronic levels that are much larger than the typical vibrational energies $\hbar \omega$. In a JT problem, the BO treatment starts with the diagonalization of (7) in the electronic degenerate space, at fixed distortion field $\vec{q}$, i.e. the diagonalization of the matrix $\Xi=\sum q_{m} V^{(m)}$. Each electronic eigenvector $\left|\psi_{\xi}\right\rangle$ of $\Xi$, of eigenvalue $\lambda_{\xi}$, generates a BO potential sheet $V_{\xi}(\vec{q})=\hbar \omega\left[\frac{1}{2} \sum_{m} q_{m}^{2}+\frac{g}{\sqrt{2}} \lambda_{\xi}(\vec{q})\right]$, including the harmonic potential of the free vibrations. At strong enough coupling $g$, the separation of the potential sheets becomes large enough that the adiabatic motion can be assumed to always follow the lowest $\mathrm{BO}$ potential sheet, while virtual electronic excitations may be treated as a small correction. For Hamiltonians of type (3), the set of points $\vec{q}_{\min }$ of minimum potential energy, i.e. the classical stable configurations, constitutes a continuous manifold, often denominated Jahn-Teller manifold (JTM), and the value of the lowest BO potential there is the classical JT stabilization energy $E_{\text {clas }}=V_{\min }\left(\vec{q}_{\min }\right)$.
On the other side, due to time-reversal invariance of $H$, the space of all possible (normalized) electronic eigenstates can be represented by an (hyper-)sphere in the $[2 L+1]$-dimensional real space (see Fig. 1). The $\mathrm{BO}$ dynamics realizes an adiabatic mapping of the vibrational space into this electronic space. [11] Indeed, every point $\vec{q}$ on the JTM (in the vibrational space) is associated to the electronic wave function $\left|\psi_{\min }(\vec{q})\right\rangle$, corresponding to the lowest eigenvalue $\lambda_{\min }$ of the electron-vibron interaction matrix $\Xi$ :

$$
\Xi(\vec{q})\left|\psi_{\min }(\vec{q})\right\rangle=\lambda_{\min }(\vec{q})\left|\psi_{\min }(\vec{q})\right\rangle .
$$

This adiabatic mapping is two-valued, since opposite points $\pm\left|\psi_{\min }(\vec{q})\right\rangle$ on the electronic sphere give the same JT stabilization energy, thus corresponding to the same optimal distortion on the JTM. This identification of the antipodal points through the mapping is the mechanism allowing the JTM to have different connectedness [12] from the electronic sphere. The latter is of course simply connected, i.e. any closed path, or loop, on it can be smoothly contracted to a single point. The JTM, instead, may well be multiply connected, i.e. it can have intrinsic "holes" in its topology. In particular, in addition to the contractable loops (such as that mapping on $\pi_{1}$ on the electronic sphere sketched in Fig. 1), on the JTM we have the nontrivial class of those loops mapped on a path going from a point to its antipode on the electronic sphere, such as $\pi_{2}$ in Fig. 1. In the traditional DJT systems $(E \otimes e, T \otimes h)$, these two classes of paths are topologically distinct: loops belonging to class 2 may never be smoothly deformed into loops belonging to class 1 , whence the multiple-connectedness property of the JTM. We see therefore that this multiple connectedness is intimately related to the mapping between the JTM and the electronic sphere. The electronic sign change characterising the loops belonging to class 2 is a case of Berry phase [9].

When also the vibrational motion is quantized, the overall (vibronic) $\mathrm{BO}$ wave function is factorized in the direct product of the electronic adiabatic state times the wave function for the slow degrees of freedom $\vec{q}$ : since the vibronic wave function is a regular, single-valued function, the $\vec{q}$ degrees of freedom must cope with the electronic phase change, which acts as a special boundary condition to quantization. As a consequence the motion on the JTM is constrained by special selection rules. For example the JTM of the simple $E \otimes e$ system is a circle: the low-energy vibronic spectrum is indeed a $j^{2}$ spectrum as for a circular rotor, but the Berry phase implies $j= \pm \frac{1}{2}, \pm \frac{3}{2}, \ldots$, instead of $j=0, \pm 1, \pm 2, \ldots$ as for an ordinary quantum rotor. [2,13] Similarly, the JTM of the $T \otimes h$ (i.e. $\mathcal{D}^{(1)} \otimes d^{(2)}$, in the spherical language) is equivalent to a sphere, [4] 14 but out of all the states, labeled by $J, M$, of a particle on a sphere, the Berry phase keeps only the odd- $J$ ones. [2, 4 14] Note in particular that in these examples the presence of a Berry phase rules out a nondegenerate ground state. The same symmetry of 
the degenerate electronic state, prior to the vibronic coupling/distortion, is enforced to the strong coupling DJT ground state.

\section{ABSENCE OF THE BERRY PHASE}

As anticipated above, though a very frequent phenomenon, the Berry phase is not automatically implied by linear JT Hamiltonians (11). The above discussion should make clear that the absence of the Berry phase in a DJT system is linked to a mechanism leading to equivalence of the paths in the class 1 and 2. This mechanism should also coexist with the two-valued adiabatic mapping sketched in the previous section. The solution of the riddle is provided by a point $\vec{q}_{d}$ on the JTM where the mapping is degenenerate, i.e. it links $\vec{q}_{d}$ not just to a pair of opposite points $\pm\left|\psi_{\min }\left(\vec{q}_{d}\right)\right\rangle$ on the electronic sphere, but to the whole circle of linear combinations $\cos \theta\left|\psi_{1}\left(\vec{q}_{d}\right)\right\rangle+\sin \theta\left|\psi_{2}\left(\vec{q}_{d}\right)\right\rangle$ of two degenerate electronic eigenstates (such as, for example, $\Delta$ in Fig. 1). If such a point is present, any loop of class 2 on the JTM may be deformed smoothly so that its image on the electronic sphere becomes half this circle, thus the single point $\vec{q}_{d}$ on the JTM: class 2 loops are therefore equivalent to class 1 (contractable) loops, and, therefore, the JTM is simply connected. No Berry phase is possible in such a case. 15]

The possibility of such degenerate points may be considered via careful analysis of the structure of the multiple $\mathrm{BO}$ potential sheets $V_{\xi}$ given by the eigenvalues $\lambda_{\xi}$ of the electron-vibration interaction operator. In most "classical" cases of Berry-phase-entangled linear JT systems $(E \otimes e, T \otimes h, \ldots)$, the lowest potential sheet remains separated from the next lowest one by a finite gap throughout the JTM. Conical intersections between these two sheets take place at some point in the distortion space, far from the potential minimum. In the $E \otimes e$, for example, such a point is the origin $\vec{q}=0$. 16] There is, however, a second possiblilty: somewhere on the JTM, the lowest $\mathrm{BO}$ potential sheet gets tangentially degenerate to the next lowest $\mathrm{BO}$ potential sheet. The energy difference between the two lowest levels is quadratic in the distance from the degenerate point.

This possibility is indeed realized in our spherical model. Take for example the case $L=2, l=2$ and consider the point $\vec{q}_{d}=\left(0,0,-\frac{1}{\sqrt{7}} g, 0,0\right)$. [7] Simple inspection of the coupling matrix $\Xi$ (8) shows that its lowest eigenvalue $-\left|q_{d}\right| \gamma_{2,-2}^{+} \equiv-\left|q_{d}\right|\langle l 0 \mid L 2, L-2\rangle=-\frac{\sqrt{2}}{7} g$ is twofold degenerate. The classical BO potential energy is $E_{\text {clas }}=V_{\min }\left(\vec{q}_{d}\right)=-\frac{1}{14} g^{2} \hbar \omega$, and no other $\vec{q}$ yields lower potential energy than this: by definition, $\vec{q}_{d}$ does belong to the JTM. By inspection of Eqs. (810), it is possible to verify that the direct coupling between the two degenerate electronic states (element $\Xi_{ \pm 2, \mp 2}$ of the coupling matrix) vanishes identically: moving away from $\vec{q}_{d}$, these two states remain degenerate to first order, and the degeneracy is only lifted by indirect, second order coupling to the other states. $\vec{q}_{d}$ is indeed a tangency point. With this, we have detailed the mechanism for the absence of a Berry phase in the $\mathcal{D}^{(2)} \otimes d^{(2)}$ model.

It is natural to search possible extensions of this mechanism to other cases of spherical DJT models (3). Eqs. (812) show that on the $\hat{q}_{0}$ axis, the electronic states are pairwise degenerate (except for the $\tilde{c}_{0}^{\dagger}|0\rangle$ state). We are interested in the pair corresponding to the lowest and next lowest BO sheet, thus to the maximum numerical value of the Clebsch-Gordan $\left|\gamma_{m,-m}^{+}\right|$. For the $\mathcal{D}^{(2)} \otimes d^{(2)}$ described above, the maximum is indeed $\gamma_{2,-2}^{+}$. 17] On the contrary, for the $\mathcal{D}^{(2)} \otimes d^{(4)}$ case, the largest coefficient is $\gamma_{0,0}^{+}=\left(\frac{18}{35}\right)^{1 / 2}$, corresponding to the singlet state. Thus on the $\hat{q}_{0}$ axis there are no degeneracy points of the two lowest BO sheets. We verified that this case has no degenerate points anywhere on the JTM, the gap between the lowest and next lowest $\mathrm{BO}$ sheet being a constant $\frac{g}{\sqrt{2}}\left|q_{d}\right|\left(\frac{5}{14}\right)^{1 / 2}=\frac{3}{14} g^{2}$ across all the JTM. We conclude that the $\mathcal{D}^{(2)} \otimes d^{(4)}$ is not a Berry-phase-free model. The same applies to all "complete" systems, i.e. the $\mathcal{D}^{(L)} \otimes d^{(l=2 L)}$, since $\langle 2 L 0 \mid L 0, L 0\rangle$ (corresponding to the nondegenerate electronic state) is the largest ClebschGordan coefficient among all $\langle 2 L \quad 0 \mid L M, L-M\rangle=$ $N(L) /(L-M) !(L+M)$ ! (where $N(L)$ is a function of $L$ only).

If we consider now the $\mathcal{D}^{(L)} \otimes d^{(l=L)}$ models, we see that the coefficients $|\langle L 0 \mid L M, L-M\rangle|$ are peaked around $|M|=\hat{M} \approx 86 \% L$ (for large $L$ ). For $L<50$, say, the peak is rather sharp, allowing to concentrate on the $2 \times 2$ block of the interaction matrix:

$$
\left(\begin{array}{cc}
\Xi_{-\hat{M},-\hat{M}} & \Xi_{-\hat{M}, \hat{M}} \\
\Xi_{\hat{M},-\hat{M}} & \Xi_{\hat{M}, \hat{M}}
\end{array}\right)=\left(\begin{array}{cc}
\chi_{\hat{M},-\hat{M}} q_{0} & 0 \\
0 & \chi_{-\hat{M}, \hat{M}} q_{0}
\end{array}\right)
$$

Other diagonal and off-diagonal contributions in the block vanish, since they could only be related to $q_{ \pm 2 \hat{M}}$, which does not exist, since $2 \hat{M}>l=L$. This block, therefore, gives a twofold degenerate electronic ground state, with second-order departures from the degeneracy, as the distortion moves away from the $\hat{q}_{0}$ axis. We conclude that the two lowest BO sheets, in all the $\mathcal{D}^{(L)} \otimes d^{(L)}$ models, get tangent at (at least) one point, thus making equivalent all loops on the JTM. As a consequence, this class of models must be considered as Berry-phase-free.

Finally, we apply the same reasoning to the study of a generic $\mathcal{D}^{(L)} \otimes d^{(l)}$ model. For $l<L$ the $|M|=\hat{M}$, at which the relevant coefficients $|\langle l \quad 0 \mid L M, L-M\rangle|$ is maximum, always satisfies the inequality $2 \hat{M}>l$. Thus, these are yet more Berry-phase-free systems. On the contrary, for $l>L$, we go from a case without $\left(\mathcal{D}^{(L)} \otimes d^{(L)}\right)$ to a case with $\left(\mathcal{D}^{(L)} \otimes d^{(2 L)}\right)$ Berry phase, thus the result is not trivial. For large enough $l$, the maximum coefficient is attained at some $\hat{M} \leq l$, and this leaves nonzero direct off-diagonal coupling elements in the $2 \times 2$ block (14). In such a case, the degenerate point is not a tangency, but a conical intersection, thus it cannot belong to the JTM. It can be verified numerically 
that the tangency point on the $\hat{q}_{0}$ axis disappears for all $l \geq l_{c}(L)$, where the "critical value" $l_{c}(L)$ is found between $L$ and $2 L$. For $L=2,3,4, \ldots 10, l_{c}(L)$ takes values 4, 4, 6, 8, 8, 10, 12, 12, 14 respectively: thus, for example, a $\mathcal{D}^{(7)} \otimes d^{(8)}$ model has no Berry phase, while $\mathcal{D}^{(9)} \otimes d^{(14)}$ can have it. Our demonstration focuses on the $\hat{q}_{0}$ axis: we cannot rule out the possibility of other tangency points elsewhere on the JTM. Thus the presence of the Berry phase is verified only for $l=2 L$, where the gap between the two lowest sheets is a constant on the JTM, while it is only likely for $l_{c}(L) \leq l<2 L$.

\section{NUMERICAL RESULTS}

We have classified Berry-phase-wise a large class of spherical DJT systems: it should be possible to evidence the signatures of this property in their vibronic spectrum. It was previously shown [7] that the ground state of the $\mathcal{D}^{(2)} \otimes d^{(2)}$ system becomes nondegenerate at strong coupling. We have applied the same technique of numerical diagonalization on a truncated basis to the next Berryphase-free model, $\mathcal{D}^{(4)} \otimes d^{(4)}$. In Fig. 2, we show the vibronic energy of the lowest $L=0$ and $L=4$ vibronic states above the $\mathrm{BO}$ potential minimum $E_{\text {clas }}$ : the lowest nondegenerate and degenerate states cross at $g \approx 8$, the former becoming the strong-coupling ground state, as the absence of a Berry phase predicts.

However, an analogous test for the $\mathcal{D}^{(4)} \otimes d^{(2)}$ model finds a degenerate $L=4$ ground state up to coupling $g=$ 20. An explanation could be searched in the nature of the lowest $L=0$ state at weak-coupling: while in $\mathcal{D}^{(L)} \otimes$ $d^{(L)}$ models it comes as a fragment of the one-vibron multiplet, in this case it originates from the two-vibron multiplet, due to angular momentum conservation. It seems as if the angular moment of the $l=2$ vibration was insufficient to screen the large electronic moment, thus, even in absence of Berry phase, the ground state remains degenerate.

\section{DISCUSSION}

In the Berry-phase-free DJT systems, the tangency of the lowest two BO sheets, strictly speaking, invalidates the BO treatment, which assumes a large gap between the lowest electronic state and the next one. Thus, paradoxically, in these systems it is not the Berry phase which is related to a breakdown of the $\mathrm{BO}$ approximation, but its absence. Indeed, we have shown that, even though these degeneracies are present only locally on the JTM, they affect radically the whole coupled dynamics.

Our analysis considers spherical DJT models: however, it can be extended to molecular point groups, by substituting the relevant Clebsch-Gordan coefficients. 18 There are cases, such as the linear $T \otimes h$ in icosahedral symmetry (or equally coupled $T \otimes(e+t)$ in cubic symmetry) which are equivalent to some spherical models, but of course this is not always the case, and other surprising results could be found by looking for tangencies in different systems.

Also, we assume a linear JT coupling scheme (Hamiltonian (1)), which is the less realistic, the stronger the JT distortion. The perturbative introduction of higher-order couplings has usually effects similar to those produced by different linear coupling $g_{E} \neq g_{T}$ in cubic symmetry, 19 i.e. of "warping" the JT trough. The continuous JTM becomes a set of isolated minima, connected by low-energy paths passing through saddle points. The symmetry of the Hamiltonian and of the JTM is reduced to the symmetry group $G$ of the molecule. Yet, the connectedness properties are topological properties, therefore they are robust against perturbations such as the potential warping: even if the symmetry is reduced, the Berry phase is still present or absent as determined by the linear part. Ham 20] has shown for the $E \otimes e$ coupling scheme that the introduction of quadratic terms in the Hamiltonian does not substantially change the picture as far as the Berry phase and the degeneracy of the ground state are concerned. In fact, even at strong JT coupling, the tunneling among rather deep isolated minima is affected by the electronic phase, [2] and, as a result, the lowest tunnel-split state retains the same symmetry and degeneracy as in the purely linear-coupling case. Of course, in the extreme limit of very large distortion, higher-order terms dominate, and the DJT is replaced by a static distortion, where any Berry phase argument becomes irrelevant.

\section{CONCLUSION}

In summary, we propose evidence for a whole family, following the $\mathcal{D}^{(2)} \otimes d^{(2)}$, of Berry-phase-free dynamical JT systems: these are the $\mathcal{D}^{(L)} \otimes d^{(l)}$ models, with $l<l_{c}(L)$, where the critical value $l_{c}(L)$ lies between $L$ and $2 L$. For these models, we also show that the absence of the Berry phase does not automatically imply a nondegenerate strong-coupling vibronic ground state. Moreover, we prove that a Berry phase is present in the $\mathcal{D}^{(L)} \otimes d^{(2 L)}$ models, which, as a consequence, have a $\mathcal{D}^{(L)}$ $[2 L+1]$-fold degenerate ground state for any coupling.

\section{ACKNOWLEDGEMENT}

We thank M. Altarelli, E. Tosatti and Lu Yu for useful discussions. 
[1] R. Englman, The Jahn Teller Effect in Molecules and Crystals (Wiley, London, 1972).

[2] I. B. Bersuker and V. Z. Polinger, Vibronic Interactions in Molecules and Crystals (Springer Verlag, Berlin, 1989).

[3] J. Ihm, Phys. Rev. B 49, 10726 (1994).

[4] A. Auerbach, N. Manini, and E. Tosatti, Phys. Rev. B 49, 12998 (1994).

[5] C. A. Mead, Rev. Mod. Phys. 64, 51 (1992).

[6] Geometric Phases In Physics, edited by A. Shapere and F. Wilczek (World Scientific, Singapore, 1989).

[7] P. De Los Rios, N. Manini and E. Tosatti, Phys. Rev. B 54, 7157 (1996).

[8] C. P. Moate, M. C. M. O'Brien, J. L. Dunn, C. A. Bates, Y. M. Liu, and V. Z. Polinger, Phys. Rev. Lett 77, 4362 (1996).

[9] M. V. Berry, Proc. R. Soc. Lond. A 392, 45 (1984).

[10] M. C. M. O'Brien, Phys. Rev. B 53, 3775 (1996).

[11] A. Ceulemans, J. Chem. Phys. 87, 5374 (1987).

[12] B. A. Dubrovin, A. T. Fomenko and S. P. Novikov, Modern Geometry: Methods and Applications; II: The Geometry and Topology of Manifolds, 2nd edition, (SpringerVerlag, Berlin, 1992).

[13] H. Koizumi and S. Sugano, J. Chem. Phys. 101, 4903 (1994).

[14] M. C. M. O’Brien, J. Phys. C 4, 2524 (1971).

[15] P. De Los Rios and N. Manini, in Recent Advances in the Chemistry and Physics of Fullerenes and Related Materials: Volume 5, edited by K. M. Kadish and R. S. Ruoff (The Electrochemical Society, Pennington, NJ, 1997), p. 468.

[16] This fact is true in general: the individual eigenvalues $\lambda_{\xi}$ of the coupling matrix are analytical (though often exceedingly complicate) expressions of the distorsion variables $\vec{q}$. Assume that very close to an hypothetical crossing point $\vec{q}_{c}$ the sheets $V_{1}$ and $V_{2}$ are the lowest and next lowest. Then, if they cross linearly, in some neighborhood of $\vec{q}_{c}$, either $V_{1}$ or $V_{2}$ gives a potential energy lower than at the crossing point. We conclude that a conical intersection cannot be a local minimum of all the BO sheets, thus it cannot belong to the JTM.

[17] Amusingly, for the $\mathcal{D}^{(2)} \otimes d^{(2)}$ system $\left|\gamma_{2,-2}^{+}\right|=\left|\gamma_{0,0}^{+}\right|$, thus opposite minimum points $\pm \vec{q}_{d}$ on the $\hat{q}_{0}$ axis have the same energy, both belong to the JTM, but one is a tangency point, and the other is not (actually it is a tangency for the upper two BO sheets). This particular feature seems accidental to the $\mathcal{D}^{(2)} \otimes d^{(2)}$, and it does not reappear in other spherical DJT systems.

[18] P. H. Butler, Point Group Symmetry Applications (Plenum, New York, 1981).

[19] M. C. M. O'Brien, Phys. Rev. 187, 407 (1969).

[20] F. S. Ham, Phys. Rev. Lett. 58, 725 (1987).

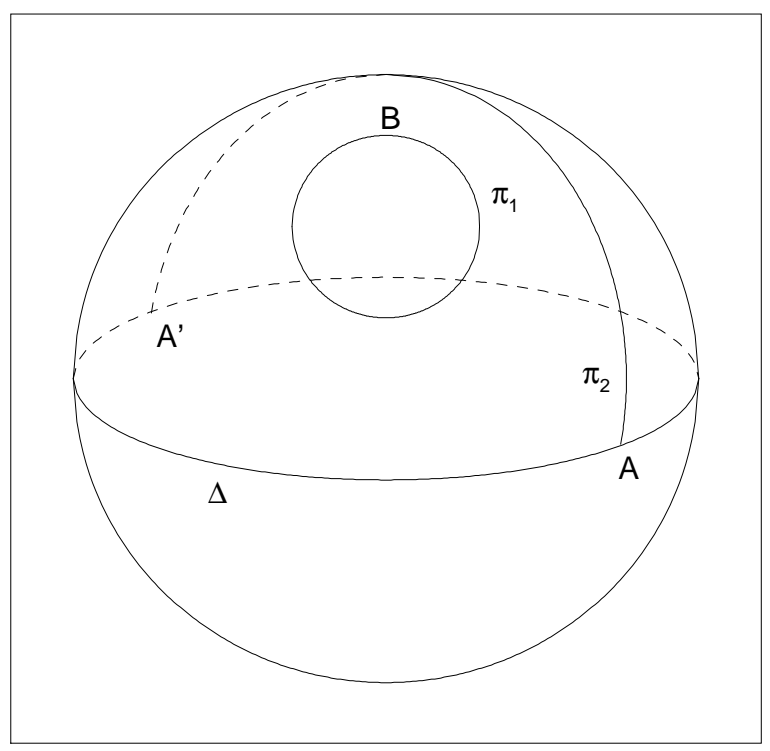

FIG. 1. A sketch of the electronic sphere. The picture individuates the two classes of paths mapping onto closed loops in the JTM: paths of the type $\pi_{2}$ involve a sign change (from A to A') of the electronic state (a Berry phase). These two types of paths can be distinguished in all $N \geq 3$-dimensional cases.

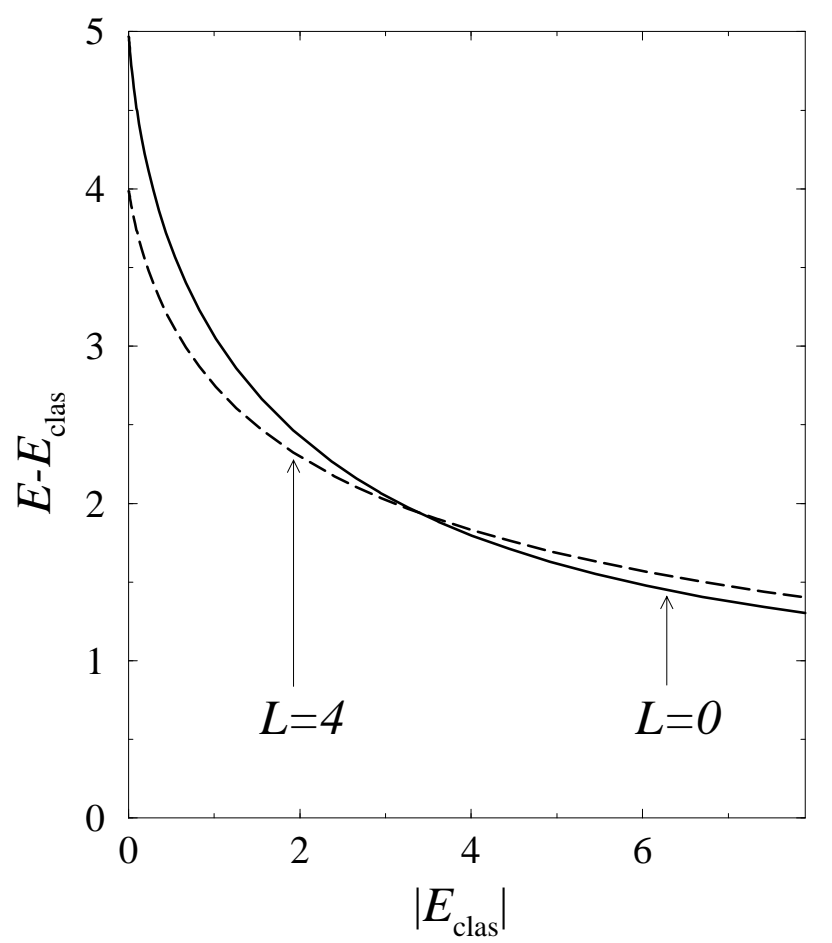


FIG. 2. The energy of the lowest $L=0$ and $L=4$ vibronic states of the $\mathcal{D}^{(4)} \otimes d^{(4)}$ system, as a function of $\left|E_{\text {clas }}\right|=\frac{63}{1144} g^{2} \hbar \omega$. The residual zero-point energy of $\frac{1}{2} \hbar \omega$ is subtracted. The energies, in units of the harmonic quantum $\hbar \omega$, are obtained by exact diagonalization of the Hamiltonian (3) on a truncated Hilbert space including up to 16 boson states, enough to reach convergence in this range of couplings. 